\title{
Another Great Pretender: A Case of Tuberculous Peritonitis Mistaken for Malignancy
}

\author{
Lauren Belak ${ }^{1}$, Jane Fazio ${ }^{2}$, Nicole Treadway ${ }^{1}$ and Iris Castro-Revoredo ${ }^{1}$ \\ ${ }^{1}$ Emory University School of Medicine, USA \\ ${ }^{2}$ David Geffen School of Medicine at UCLA, USA
}

*Corresponding author: Lauren Belak, Emory University School of Medicine, 100 Woodruff Circle, Atlanta, GA, 30322, USA,E-mail: Ibelak@emory.edu

\begin{abstract}
Background: Tuberculosis (TB) is the leading cause of death from a single infectious agent worldwide. Extrapulmonary TB accounts for nearly a third of cases, with the prevalence of abdominal TB steadily rising. However, such disease poses a significant diagnostic challenge due to lack of pathognomonic findings and sensitive testing modalities, such that nearly $40 \%$ of cases are initially missed. We present a case of peritoneal TB that closely resembled malignancy.
\end{abstract}

Case Presentation: A 34-year-old female with a history of anemia, low grade cervical dysplasia, and high-risk HPV presented with a 7-day history of worsening abdominal distension, following one month of progressive fatigue, anorexia, weight loss, and subjective fevers. She denied respiratory symptoms, night sweats or changes in bladder or bowel function. The patient immigrated from Mexico 15 years prior, and briefly lived with her father and brother who were both treated for TB decades before. Her aunt and grandmother died of uterine cancer. Her physical exam was notable for a distended abdomen with diffuse tenderness to palpation. Computed tomography (CT) and magnetic resonance imaging (MRI) revealed ascites with peritoneal thickening and an adnexal cyst. Chest CT showed scattered centrilobular pulmonary nodules and axillary lymphadenopathy. Further workup yielded an elevated Ca-125, and serially negative sputum smears for acid fast bacillus (AFB). Sequential diagnostic paracenteses revealed elevated polymorphonuclear cells, but negative cytology, gram stains and AFB stains. The patient was discharged, although returned with similar symptoms one month later. Ultimately, exploratory laparoscopy revealed diffuse milliary peritoneal implants with biopsy positive for noncaseating granulomas and AFB. Rifampin, isoniazid, pyrazinamide and ethambutol were initiated for treatment of peritoneal TB.
Conclusions: Reaching the final diagnosis in this case was challenging because TB was not highest on the differential, as the peritoneal implants, ovarian cyst, and elevated tumor markers were most concerning for malignancy. Furthermore, delay in diagnosis occurred because all minimally invasive tests were inconclusive. A high index of suspicion for peritoneal TB must be maintained in patients who present with ascites, even in the absence of respiratory symptoms, predisposing comorbidities, or identification of mycobacteria in bodily fluid. A lower threshold for peritoneal biopsy with exploratory laparoscopy is also warranted in such cases for earlier diagnosis and life-saving medical management.

\section{Keywords}

Mycobacteria tuberculosis, Peritoneal tuberculosis, Abdominal tuberculosis, Ovarian malignancy

\begin{abstract}
Abbreviations
TB: Tuberculosis; CT: Computed Tomography; MRI: Magnetic Resonance Imaging; AFB: Acid-Fast Bacilli, PCR: Polymerase Chain Reaction; IGRAs: Interferon Gamma Release Assays; CIN-1: Cervical Intraepithelial Neoplasia; ASC-H: Atypical Squamous Cells- cannot exclude HSIL; ASCUS: Atypical Squamous Cells of Undetermined Significance; HPV: Human Papillomavirus; Hgb: Hemoglobin; MCV: Mean Corpuscular Volume; Hgb: Hemoglobin; MCV: Mean Corpuscular Volume; ADA: Adenosine Deaminase.
\end{abstract}

\section{Introduction}

Tuberculosis (TB) is a communicable disease caused by the bacteria Mycobacterium tuberculosis, and is the leading cause of death from a single infectious agent worldwide [1]. A quarter of the world's population

Citation: Belak L, Fazio J, Treadway N, Revoredo IC (2021) Another Great Pretender: A Case of Tuberculous Peritonitis Mistaken for Malignancy. Clin Med Rev Case Rep 8:352. doi.org/10.23937/23783656/1410352

Accepted: June 26, 2021: Published: June 28, 2021

Copyright: (c) 2021 Belak L, et al. This is an open-access article distributed under the terms of the Creative Commons Attribution License, which permits unrestricted use, distribution, and reproduction in any medium, provided the original author and source are credited. 
remains infected as drug resistant strains continue to emerge [1]. Although the majority of identified cases involve the respiratory system, rates of abdominal TB are increasing internationally. Now the sixth most common cause of extrapulmonary disease, incidence of abdominal TB has increased due in part to rising immunocompromised states, including HIV, alcoholic liver disease, diabetes and renal disease [2,3].

Perhaps most concerning about this illness is how difficult it can be to clinically diagnose. TB may infect single organs (without respiratory involvement) that may symptomatically and radiographically mimic pancreatic, colonic, ovarian, peritoneal, or lymphatic malignancy. In these cases, accurate clinical diagnosis is only made in half of affected patients [4]. Patients may also present with vague complaints, including abdominal pain, weight loss, and distention [5], which can mimic more common chronic liver diseases, HIV and abdominal cancers [6]. Even more challenging is the paucity of highly sensitive and specific testing despite numerous available diagnostic modalities. For instance, acid-fast bacilli and Ziehl-Neelsen staining of peritoneal fluid carries a sensitivity of less than $5 \%$, while the sensitivity of bacterial culture of ascitic fluid ranges from $21-35 \%[7,8]$. Gene amplification techniques such as polymerase chain reaction (PCR) assays for bacteria may be employed when AFB testing is negative, and while they show promise for ruling in extrapulmonary $T B$, there is marked variability in sensitivities across tissue samples $[9,10]$. Although interferon gamma release assays (IGRAs), which measure levels of interferon released by $T$ cells in response to TB antigens, are able to confirm a patient's exposure to $T B$, they are incapable of differentiating between active and latent infection [5]. Furthermore, meta-analysis of quantiferon gold, a common form of IGRA, carries a sensitivity of only $72 \%$ for extrapulmonary TB [11].

Given these diagnostic limitations and the rise of abdominal TB worldwide, it is critical for providers to maintain a high index of suspicion for this diagnosis when a patient presents with otherwise inconclusive testing and nonspecific physical and laboratory findings. Here we describe a case of peritoneal TB that closely resembled malignancy in a patient with a history of cervical intraepithelial neoplasia grade I and highrisk HPV. Mycobacterial PCR, AFB, and cytology of the patient's bodily fluids were all negative for mycobacteria, and the patient lacked underlying predisposing comorbidities or a history of respiratory symptoms. Our vignette illustrates how abdominal TB may mimic intra-abdominal carcinomatosis, emphasizes the subtle though salient differences in clinical presentation, and illuminates the important role of peritoneal biopsy to distinguish between diagnoses. Given that abdominal TB has a mortality rate as high as $20 \%$ [12-14], efforts to improve early diagnosis and medical management are essential.

\section{Case Description}

A 34 year old Spanish-speaking female with a past medical history of anemia, multiple chlamydial infections, and abnormal pap smears (Cervical intraepithelial neoplasia (CIN-1), Atypical squamous cells- cannot exclude HSIL (ASC-H), and atypical squamous cells of undetermined significance (ASCUS) with high risk human papillomavirus (HPV)) was admitted to the hospital with a 7-day history of worsening abdominal distention in the setting of a month of progressive fatigue, intermittent fevers, limited oral intake, nausea and a five-pound weight loss. She denied any respiratory symptoms, night sweats, or changes in bladder or bowel function, and her last menstrual period was one week prior. She had no recent travel history or sick contacts. The patient had presented to the Emergency Department a month prior for non-radiating, right sided abdominal pain and was found to have nonspecific trace perihepatic free fluid on ultrasound. The remainder of her previous workup was unremarkable. She was fluid resuscitated and discharged with instructions to return if her symptoms worsened.

The patient was originally from Mexico, and moved to the United States with her family at the age of 19 . She came to America to work as a construction worker, and had not been back since. For one year at the age of 23, she reports living with her father, who was incidentally found to have active tuberculosis and subsequently deported to Mexico. Her brother was also diagnosed with tuberculosis 20 years prior. Her family history was otherwise significant for fatal uterine malignancy in her paternal grandmother and aunt. She reported smoking 1-2 cigarettes a week over the prior 4 years, and was in a monogamous relationship with one male partner with whom she was sexually active for three years. She denied any history of alcohol or intravenous drug use or homelessness, but had once spent several nights in jail years prior.

Upon inpatient admission, her vitals were within normal limits, and physical exam was notable only for a distended, diffusely tender abdomen most prominent in the right upper quadrant. Murphy's sign was negative and no fluid wave was present. Pregnancy test was also negative. Laboratory reports were notable for a stable microcytic anemia (Hemoglobin (Hgb) 11.2, Mean Corpuscular Volume (MCV) (80), Thrombocytosis (373), and urinary analysis with 1+ proteinuria and 3-10 red blood cells. The remaining laboratory workup did not reveal any electrolyte abnormalities, or renal or hepatic dysfunction. Initial abdominal CT Abdomen/ Pelvis with IV contrast revealed small to moderate volume ascites with mild enhancement along the right peritoneal wall, a loculated $1.5 \times 3.6 \times 7.7 \mathrm{~cm}$ pocket of fluid along the anterior liver, and an ill-defined right adnexal cystic structure $3 \mathrm{~cm}$ in size (Figure 1). Thickening of the peritoneum with anterior nodules also raised suspicion for peritoneal carcinomatosis, 


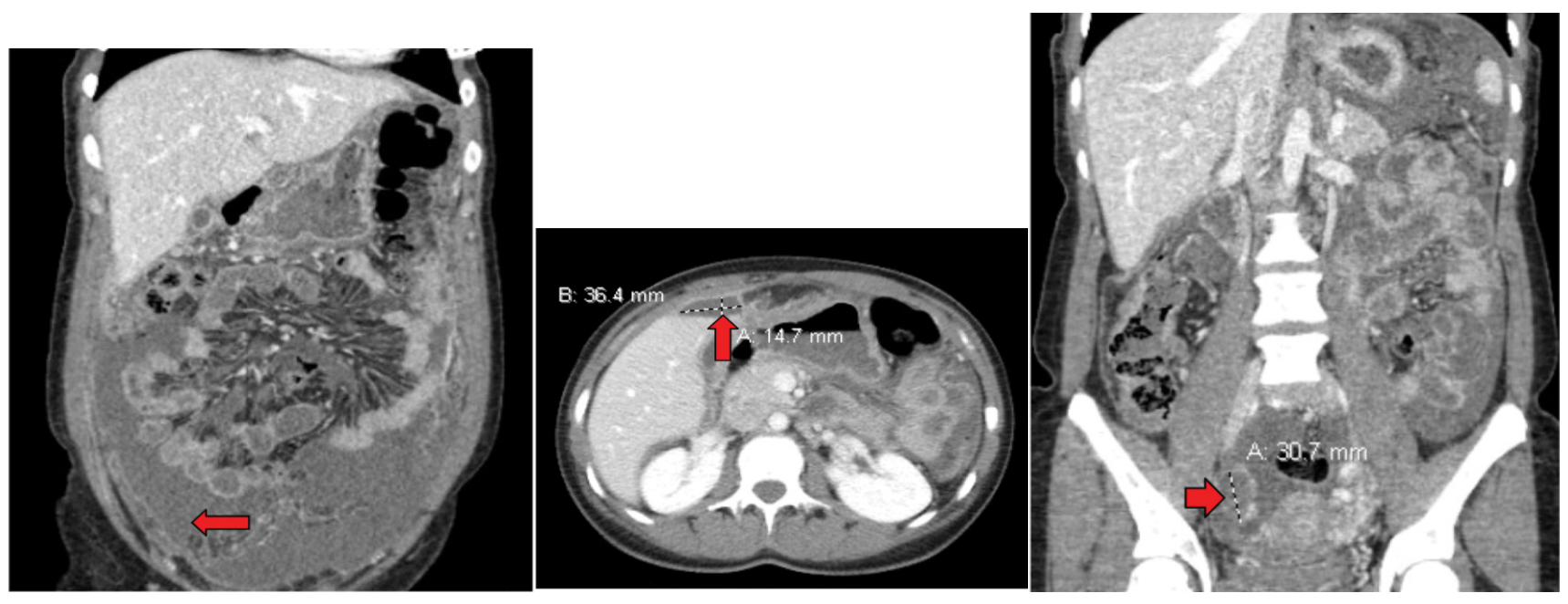

Figure 1: Initial Abdominal CT with IV contrast. Coronal view (left) revealing thickening of the peritoneum with nodularities anteriorly and ascites. Axial view (middle) shows loculated fluid collection anterior to the liver. Coronal view (right) of $3.0 \mathrm{~cm}$ right adnexal cystic structure.

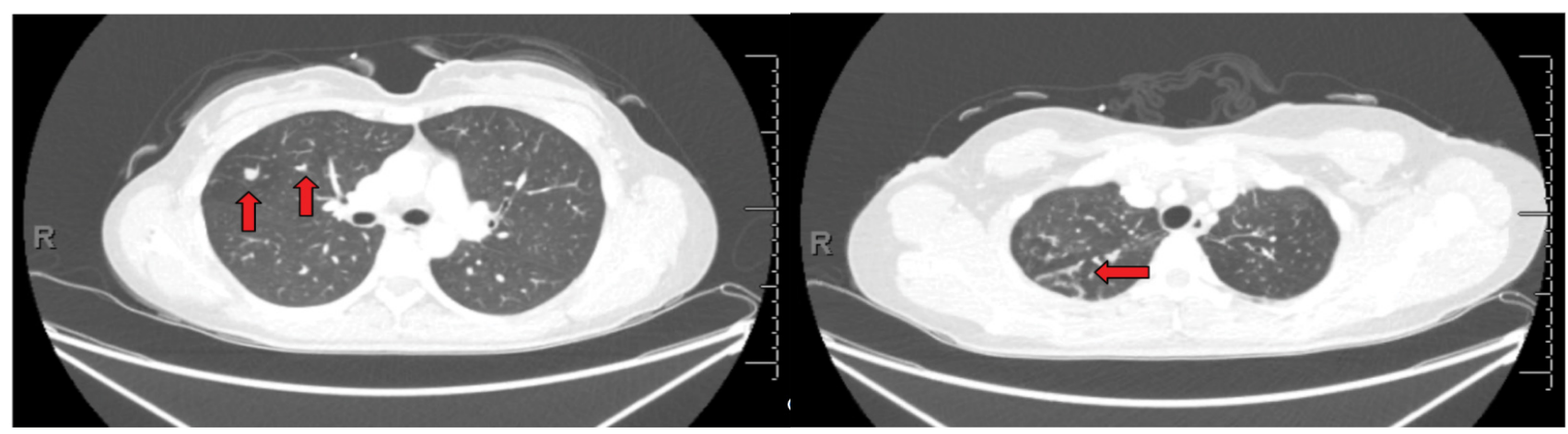

Figure 2: Chest CT revealing scattered pulmonary nodules exclusively in the bilateral upper lobes, right greater than left. The 2 largest modules are seen in the right upper lobe (left image) and measure $0.8 \mathrm{~cm}$ (left) and $0.9 \mathrm{~cm}$ (right). There is associated bronchial wall thickening and tree-in-bud nodularity (right image).

possibly associated with reactive intra-abdominal and pelvic ascites or disseminated malignancy. Subsequent Magnetic Resonance Imaging (MRI) of the abdomen with and without contrast additionally revealed a small endometrioma versus hemorrhagic cyst in the left ovary, large volume ascites, and peritoneal nodularity and thickening. Echinococcus antibody was negative, HIV test was negative, and Hepatitis A, B and C serologies were without active disease.

Chest CT was notable for scattered sub centimeter centrilobular nodules, mucoid impaction, and tree-inbud pattern exclusively in the upper lobes, which raised concern for infection, particularly mycobacterium tuberculosis or atypical mycobacterium (Figure 2). Multiple subcarinal and right hilar lymph nodes were also appreciated, which were thought to reflect intraabdominal pathology (Figure 3). The patient was also found to have prominent axillary lymphadenopathy and a possible right breast mass on the third day of hospitalization. The Gynecologic Oncology team was consulted for further assessment of underlying gynecologic malignancy. Additional testing for metastatic breast cancer markers revealed an elevated ovarian cancer marker, Ca-125 (630) and mildly elevated breast cancer marker, CA 27-29 (45). Other markers of gastrointestinal, pancreatic, and breast disease, including CEA, CA 19-9, CA 15-3 were normal. Prolactin was also normal. A follow up diagnostic mammogram did not show any evidence of malignancy (BIRADS1).

Over the course of her admission, the patient received three sequential diagnostic paracenteses notable for a lymphocytic pleocytosis $169-72 \%$ lymphocytes, $8 \%-16 \%$ neutrophils/bands body fluid, 4\%-12\% macrophages, 1340-2013 WBC/mcL body fluid), but consistently negative cytology, gram stain, culture and AFB stains. Blood cultures and two sputum samples were negative for acid-fast bacilli or any other bacterial growth. Mycobacterial PCR from a sputum sample was also negative. The patient was discharged in stable condition to follow up with her primary care physician and gynecologic-oncologic providers, with the etiology of her ascites and peritoneal implants still unknown.

The patient presented to the Emergency Department one month later with yeast-like vaginal discharge and cervical motion tenderness, and was treated 


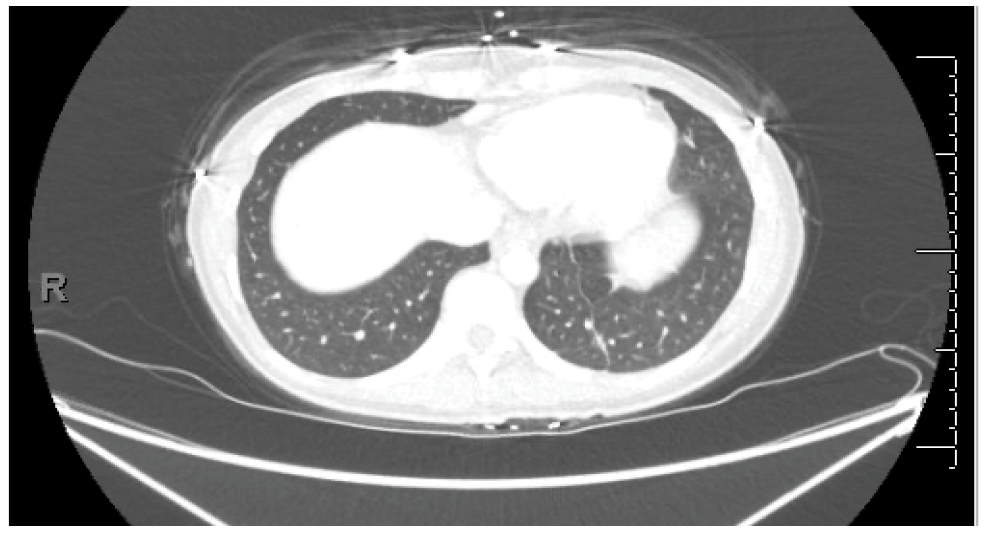

Figure 3: Prominent subcarinal and right hilar lymph nodes, with multiple prominent cardiophrenic lymph nodes measuring up to $0.7 \mathrm{~cm}$ in short axis, thought to be indicative of intra-abdominal pathology.

for a suspected sexually transmitted infection with doxycycline and fluconazole. For the week prior, she experienced progressively worsening lower abdominal pain and fullness, as well as decreased appetite and intermittent shortness of breath. She denied fevers, chills, chest pain, cough, nausea or vomiting. She was subsequently admitted to the hospital for abdominal pain with concern that her worsening symptoms could be due to progression of malignancy.

Upon admission, her vitals were within normal limits, and her exam was notable for right upper quadrant tenderness without rebound or guarding. Murphy's sign was negative. Complete Blood Count (CBC) and Complete Metabolic Panel (CMP) results were within the normal range. A CT Abdomen redemonstrated a $3 \mathrm{~cm}$ right ovarian cyst, ascites, and thickening of the peritoneum with multifocal nodularity, unchanged from her prior hospitalization. The patient was again discharged in stable condition, but given that the etiology of her ascites and peritoneal implants remained unclear, she was scheduled for a diagnostic laparoscopy the following week.

Intraoperatively, diffuse milliary-appearing peritoneal implants were found, involving the anterior abdominal wall, right upper quadrant, liver surface, hemidiaphragm, appendiceal serosa, small and large bowel mesentery, uterus, right fallopian tube, and ovaries. The extensive nature of the disease was unresectable, and no surgical intervention was completed. Peritoneal biopsy revealed multiple caseating and noncaseating granulomas with giant cells admixed with few granulomas containing small areas of caseous necrosis. AFB staining revealed rare acidfast positive bacilli, and cytology was otherwise negative for malignancy. The patient was subsequently hospitalized for urogenital and peritoneal tuberculosis, and initiated on a 9-month course of oral rifampin $600 \mathrm{mg}$, isoniazid $300 \mathrm{mg}$, pyrazinamide $1000 \mathrm{mg}$, and ethambutol $800 \mathrm{mg}$ therapy. Two subsequent sputum cultures were negative and Chest X-ray remained clear. Following treatment, the patient continues to do well and has subsequently remained asymptomatic.

\section{Conclusion}

Diagnosing abdominal TB remains one of the great challenges in medicine, as cost-effective and accurate testing modalities for extrapulmonary manifestations are lacking across the globe. At present, ultrasound and CT scan may reveal generalized or localized ascites with thickening of the peritoneum and lymphadenopathy, as were seen in our case, yet such results lack specificity [15-19]. Direct staining for acid-fast bacilli from bodily fluids is also positive in fewer than $5 \%$ of cases [20]. Ascitic fluid can be cultured to increase diagnostic yield $[21,22]$, although sensitivity of mycobacterial culture is low (21-35\%) [23], and mortality is high among patients waiting weeks for their results [22]. Serial cultures, AFB, and smears from bodily fluid may also be persistently negative for mycobacteria, and no histopathologic or bacteriological finding is the gold standard for diagnosis alone $[20,24]$. The value of the aforementioned tests for diagnosing tuberculous ascites remains limited.

Our case directly reflects these unfortunate realities, and reaching the final diagnosis was even more challenging because TBwasnothighest on the differential. Our patient not only lacked pulmonary symptoms or the underlying comorbidities that predispose patients to abdominal TB (such as immunosuppression, diabetes and renal disease), but her peritoneal implants, ovarian cyst, and elevated Ca-125 were more concerning for malignancy. Non-specific symptoms, radiographic mimicry with abdominal malignancy, and significant limitations in the sensitivities of direct AFB staining, mycobacterial cultures, and quantiferon gold assays erroneously guided our diagnostic workup with further imaging, cytologic analysis, and scheduled oncology outpatient follow up, which ultimately resulted in rehospitalization and delay in treatment.

In hindsight, subtle features of this case could have helped clinicians distinguish peritoneal TB from underlying peritoneal and ovarian malignancy. Prior research suggests female patients with peritoneal TB are younger (median age of 39 vs. 63), have a lower Ca- 
125 level ( $448 \mathrm{U} / \mathrm{mL}$ vs. $1848 \mathrm{U} / \mathrm{mL}$ ) and significantly less severe omental and mesenteric involvement relative to their counterparts with peritoneal carcinoma [25]. While our test for ascitic fluid adenosine deaminase (ADA) activity was not successfully completed, a recent study found that ascitic ADA levels in individuals with tuberculous peritonitis and peritoneal carcinomatosis were $66.76 \pm 32.09 \mathrm{IU} / \mathrm{L}$ and $13.89 \pm 8.95 \mathrm{IU} / \mathrm{L}$, respectively $(P<0.01)$ [26], suggesting that ADA analysis can help distinguish the two. In spite of this, our patient's ascitic fluid lymphocytic pleocytosis (68-72\% lymphocytes) should have also heightened suspicion, as this is an indicator of chronic tuberculous infections and has been observed in other cases of TB peritonitis $[27,28]$. Furthermore, our patient's tobacco uses at baseline increased her risk of tuberculosis [1], while her brief incarceration and shared residence with afflicted family members greatly heightened her likelihood of exposure.

Given such salient findings and otherwise inconclusive diagnostic testing, a lower threshold for peritoneal biopsy is warranted in patients that present similarly. Our patient did not undergo exploratory laparoscopy until nearly three months after presentation, at which point her disease was so extensive that it was deemed unresectable. Due to diagnostic lag, up to $40 \%$ of patients with abdominal tuberculosis may be missed and initially present with advanced findings that require surgical management [29]. Perforations, abscesses, fistulas and bowel obstruction are just some of the many surgical emergencies that could be avoidable, especially because abdominal TB is largely responsive to medical treatment and carries a favorable prognosis [30,31]. Laparoscopy is now employed more frequently for diagnosis [20], as it is minimally-invasive, safe, timeefficient, and carries more than $75 \%$ accuracy for tuberculous peritonitis [32,33]. Based on outcomes of over 1000 cases of tuberculous peritonitis, researchers have also supported exploratory laparoscopy and peritoneal biopsy for more prompt diagnosis and treatment [34]. Our case directly reveals the value of an aggressive diagnostic approach, and should provide clinicians with the confidence to consider this step, especially when other testing modalities are inconclusive.

In conclusion, diagnosing peritoneal TB remains an international public health concern due to a lack of pathognomonic findings and sensitive testing modalities. Clinicians must be cognizant of the challenges encountered in diagnosing this condition, and maintain a high index of suspicion when patients present with ascites, even in the absence of respiratory symptoms, predisposing comorbidities, or identification of mycobacteria in bodily fluid. As a medical community, we must challenge long-standing misconceptions that abdominal TB is rare and associated with active pulmonary symptoms [35]. Ending the TB epidemic by 2030 is a target of the World Health Organization, however researchers suggest that current rates of incidence decline must be doubled to reach corresponding 2020 milestones (WHO). Implementing a lower threshold for peritoneal biopsy among patients that present with ascites and unrevealing workups may help achieve this goal, and as our case depicts, may be warranted for earlier diagnosis and life-saving medical management.

\section{Acknowledgement}

The authors have no conflicts of interest or financial supporters to disclose. We would like to thank our patient, her family, Grady Hospital, and Emory University School of Medicine for graciously contributing to this case report. All authors contributed to the production of this case report.

\section{References}

1. World Health Organization (2019) Global tuberculosis report.

2. Farías llamas OA, López ramírez MK, Morales amezcua JM, Medina Quintana M, Buonocunto Vázquez G, et al. (2005) Peritoneal and intestinal tuberculosis: An ancestral disease that poses new challenges in the technological era. Case report and review of the literature. Rev Gastroenterol Mex 70: 169-179.

3. Centers for Disease Control and Prevention (2016) Reported Tuberculosis in the United States.

4. Kapoor VK, Sharma LK (1988) Abdominal tuberculosis. Br J Surg 75: 2-3.

5. Wu DC, Averbukh LD, Wu GY (2019) Diagnostic and Therapeutic Strategies for Peritoneal Tuberculosis: A Review. J Clin Transl Hepatol 7: 140-148.

6. Bulut Gökten D, Katipoglu B, Basara E, Ates I, Yılmaz N (2018) A case report of peritoneal tuberculosis: A challenging diagnosis. Case Reports in Infectious Diseases: 1-3.

7. Manohar A, Simjee AE, Haffejee AA, Pettengell KE (1990) Symptoms and investigative findings in 145 patients with tuberculous peritonitis diagnosed by peritoneoscopy and biopsy over a five-year period. Gut 31: 1130-1132.

8. Fei G-J, Zhang L-F, Shu H-J (2018) [values of different laboratory diagnostic approaches for tuberculous peritonitis]. Zhongguo Yi Xue Ke Xue Yuan Xue Bao 40: 534-538.

9. Denkinger CM, Schumacher SG, Boehme CC, Dendukuri N, Pai M, et al. (2014) Xpert MTB/RIF assay for the diagnosis of extrapulmonary tuberculosis: A systematic review and meta-analysis. Eur Respir J 44: 435-446.

10. Kohli M, Schiller I, Dendukuri N, Dheda K, Denkinger CM, et al. (2018) Xpert $囚$ MTB/RIF assay for extrapulmonary tuberculosis and rifampicin resistance. Cochrane Database Syst Rev 8: CD012768.

11. Fan L, Chen Z, Hao X-H, Hu Z-Y, Xiao H-P (2012) Interferongamma release assays for the diagnosis of extrapulmonary tuberculosis: A systematic review and meta-analysis. FEMS Immunol Med Microbiol 65: 456-466.

12. Chalya PL, McHembe MD, Mshana SE, Rambau PF, Jaka $\mathrm{H}$, et al. (2013) Clinicopathological profile and surgical 
treatment of abdominal tuberculosis: A single Centre experience in northwestern Tanzania. BMC Infect Dis 13: 270.

13. Chou CH, Ho MW, Ho CM, Lin PC, Weng CY, et al. (2010) Abdominal tuberculosis in adult: 10-year experience in a teaching hospital in Central Taiwan. J Microbiol Immuno Infect 43: 395-400.

14. Chen HL, Wu MS, Chang WH, Shih SC, Chi H, et al. (2009) Abdominal tuberculosis in southeastern Taiwan: 20 years of experience. J Formos Med Assoc 108: 195-201.

15. Rathi P, Gambhire P (2016) Abdominal Tuberculosis. J Assoc Physicians India 64: 38-47.

16. Sheikh M, Abu-Zidan F, M al-Hilaly M, Behbehani A (1995) Abdominal tuberculosis: Comparison of sonography and computed tomography. J Clin Ultrasound 23: 413-417.

17. Akhan O, Pringot J (2002) Imaging of abdominal tuberculosis. Eur Radiol 12: 312-323.

18. Batra A, Gulati MS, Sarma D, Paul SB (2000) Sonographic appearances in abdominal tuberculosis. J Clin Ultrasound 28: $233-245$

19. Maclean KA, Becker AK, Chang SD, Harris AC (2013) Extrapulmonary tuberculosis: Imaging features beyond the chest. Can Assoc Radiol J 64: 319-324.

20. Weledji EP, Pokam BT (2017) Abdominal tuberculosis: Is there a role for surgery? World J Gastrointest Surg 9: 174181.

21. Rana S, Farooqui MR, Rana S, Anees A, Ahmad Z, et al. (2015) The role of laboratory investigations in evaluating abdominal tuberculosis. J Family Community Med 22: 152157.

22. Chow KM, Chow VC, Hung LC, Wong SM, Szeto CC (2002) Tuberculous peritonitis-associated mortality is high among patients waiting for the results of mycobacterial cultures of ascitic fluid samples. Clin Infect Dis 35: 409-413.

23. Huang L-L, Xia HH-X, Zhu S-L (2014) Ascitic fluid analysis in the differential diagnosis of ascites: Focus on cirrhotic ascites. J Clin Transl Hepatol 2: 58-64.

24. Evans RP, Mourad MM, Dvorkin L, Bramhall SR (2016) Hepatic and intra-abdominal tuberculosis: 2016 update. Curr Infect Dis Rep 18: 45.
25. Choi CH, Kim CJ, Lee YY, Kim JS, Song T, et al. (2010) Peritoneal tuberculosis: A retrospective review of 20 cases and comparison with primary peritoneal carcinoma. Int $\mathrm{J}$ Gynecol Cancer 20: 798-803.

26. Kang SJ, Kim JW, Baek JH, Kim SH, Kim BG, et al. (2012) Role of ascites adenosine deaminase in differentiating between tuberculous peritonitis and peritoneal carcinomatosis. World J Gastroenterol 18: 2837-2843.

27. Brown JD, Dac An N (1976) Tuberculous peritonitis. Low ascitic fluid glucose concentration as a diagnostic aid. Am J Gastroenterol 66: 277-282.

28. Aggarwal G, Grover M, Kamath P (2010) Where is the Bacillus: A Case of Tuberculous Peritonitis. Am J Gastroenterol 105: 266.

29. Saxena P, Saxena S (2016) The role of laparoscopy in diagnosis of abdominal tuberculosis. Int Surg J 3:15571563.

30. Harries AD (1990) Tuberculosis and human immunodeficiency virus infection in developing countries. Lancet 335: 387-390.

31. Rai S, Thomas WM (2003) Diagnosis of abdominal tuberculosis: The importance of laparoscopy. J R Soc Med 96: 586-588

32. Fillion A, Ortega-Deballon $\mathrm{P}, \mathrm{Al}$-Samman $\mathrm{S}$, Briault $\mathrm{A}$, Brigand C, et al. (2016) Abdominal tuberculosis in a low prevalence country. Med Mal Infect 46: 140-145.

33. Târcoveanu E, Dimofte G, Bradea C, Lupaşcu C, Moldovanu $\mathrm{R}$, et al. (2009) Peritoneal tuberculosis in laparoscopic era. Acta Chir Belg 109: 65-70.

34. Chow KM, Chow VC, Szeto CC (2003) Indication for peritoneal biopsy in tuberculous peritonitis. Am J Surg 185: 567-573.

35. Abu-zidan FM, Sheek-hussein M (2019) Diagnosis of abdominal tuberculosis: Lessons learned over 30 years: Pectoral assay. World J Emerg Surg 14: 33. 